\title{
A Survey of all Existing Clustering Protocols in VANETS but Main Emphasis of Survey Laid on Currently using Protocol i.e TCDGP
}

\author{
Jyotsna Rao Dawande \\ Student, Dept. of CSE \\ University Institute of \\ Technology-RGPV, Bhopal, \\ Madhya Pradesh
}

\author{
Sanjay Silakari, Ph.D \\ HoD, Dept. of CSE \\ University Institute of \\ Technology-RGPV, Bhopal, \\ Madhya Pradesh
}

\author{
Anjna Deen \\ Professor, Dept. of CSE \\ University Institute of \\ Technology-RGPV, Bhopal, \\ Madhya Pradesh
}

\begin{abstract}
In recent years VANets have come up as new information promulgation technology. To enhance vehicle and road safety, traffic efficiency, and ease as well as comfort to both drivers and passengers, VANets become an active area of research. In VANets, vehicle work as a node. Vehicle collect different types of information like road traffic and environmental information and transmit them to intended entities but it become a challenging task to route the information to destination because of sparse distribution and high mobility of vehicles in road. To address this issue, clustering has been widely used in many existing proposals in articles. In this paper various challenges for clustering in VANets are discussed briefly but main emphasis laid on the current technique which is widely used nowadays i.e Token-based Clustered data Gathering Protocol (TCDGP).
\end{abstract}

\section{Keywords:}

VANets, TCDGP, RSUs, V2V, V2R, CH.

\section{INTRODUCTION}

Vehicular ad hoc Network (VANets) is a rising new technology integrating ad hoc network, wireless local area network (WLAN) and cellular technology to achieve intelligent inter vehicle communications and improve road traffic safety and efficiency.

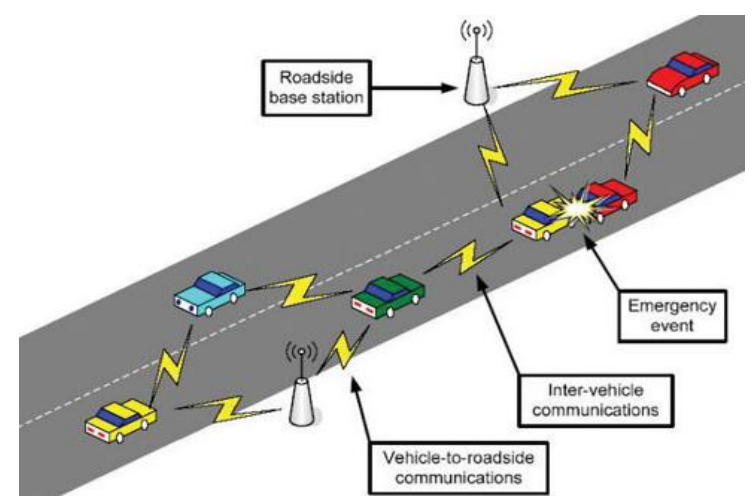

Figure 1: Vehicular Ad Hoc Network Overview

\subsection{Overview of VANeTs}

Intelligent Transportation Systems (ITSs): In Intelligent transportation systems, every vehicle takes on the role of sender, receiver, and router [1] to broadcast information to the vehicular network or transportation office, which then uses the knowledge to make sure safe and free-flow of traffic. For communication between vehicles and Road side Units (RSUs), vehicles should be equipped with some sort of radio interface or On Board Unit (OBU) that allows short-range wireless ad hoc networks to be formed [2]. Vehicles should also be fitted with hardware that allows detailed position information like global Positioning System (GPS) or a Differential global Positioning System (DGPS) receiver. Fixed roadside units, which are connected to the backbone network, should be in place to facilitate communication. The number and distribution of RSUs depends on the used communication protocols. For e.g., some protocols depend upon RSUs to be distributed uniformly throughout the whole road network; some require roadside units only at intersections, while others require RSUs only at region borders. Figures 1, 2 and 3 represents the possible communication configurations in ITS. These include intervehicle, V2R, and routing-based communications.

\subsubsection{Inter-Vehicle Communication}

In this configuration (Fig. 2) uses multi-hop multicast/broadcast to send traffic related information over multiple hops to a group of receivers. In ITS, vehicles only need to be take care of activity on the road ahead and not behind.

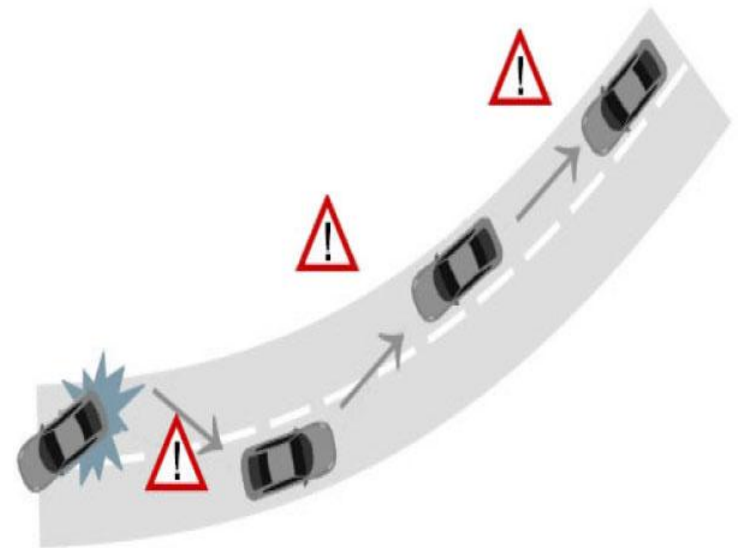

Fig. 2: Inter-Vehicle Communication

\subsubsection{Vehicle-to-Roadside Communication}

The vehicle-to-roadside communication configuration (Fig. 3) represents a single hop broadcast where the RSUs send a broadcast message to all equipped vehicles in the vicinity.

V2R communication configuration provides a high bandwidth link between vehicles and roadside units. The RSUs may be placed in every kilometer or less, allowing high data rates to be maintained in heavy traffic.

\subsubsection{Routing-Based Communication}

This configuration (Fig. 4) is a multi-hop unicast in which a message is propagated in a multi- hop fashion until the vehicle carrying the desired data is reached. 


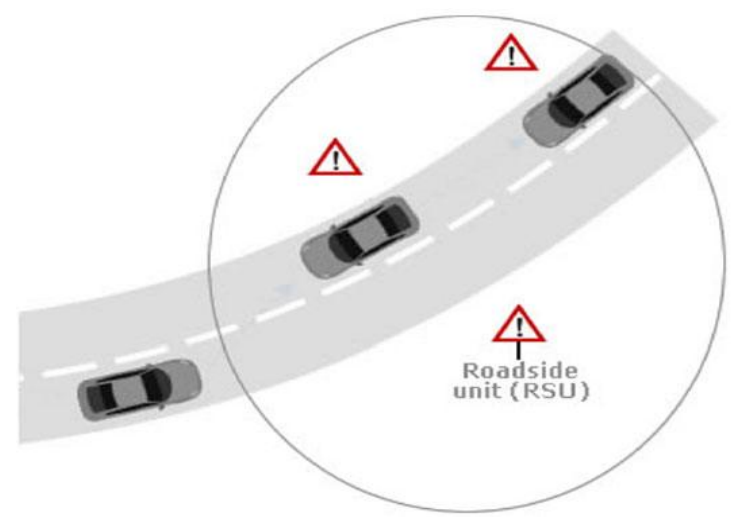

Fig. 3: Vehicle-to-Roadside Communication

When vehicle is received a query posses the expected piece of information, the application at that vehicle immediately sends a unicast message containing the information to the vehicle from which it received the request and then charged with the job of forwarding it towards the query source.

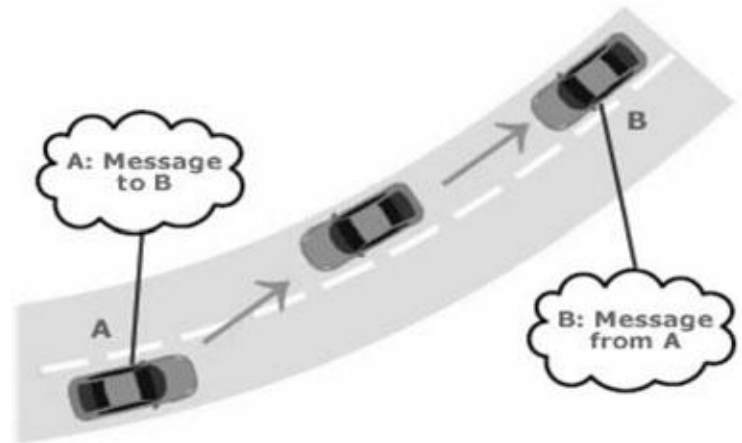

Fig. 4: Routing-Based Communication

\section{STANDARDS: WIRELESS ACCESS IN VANETS}

Product development becomes very simple due to standards. Standards simplify product development, helps in cost reduction, and enable users to compare competing products. Interconnectivity and interoperability can be achieved only due to the use standards and to enable the rapid implementation of new technologies, verification of emergence of new product should be done. Only through the use of standards can the requirements of interconnectivity and interoperability be guaranteed and the emergence of new products is verified to enable the rapid implementation of new technologies. There are various standards that relate to wireless access in vehicular environments.

\subsection{Dedicated Short Range Communication (DSRC)}

Dedicated Short Range Communications (DSRC) is a short to medium range communications service that was developed to support $\mathrm{V} 2 \mathrm{~V}$ and $\mathrm{V} 2 \mathrm{R}$ communications. A wide range of applications, including $\mathrm{V} 2 \mathrm{~V}$ safety messages, information of traffic, toll collection, drive-through payment, and many others are covered by such communication. The goal of DSRC is providing high data transfers and low communication latency in small communication zones.

\subsection{IEEE 1609-standards for wireless access in vehicular environments (WAVE) (IEEE 802.11p)}

Wireless connectivity between moving vehicles can be provided by existing 802.11a compliant devices with data rates of up to $54 \mathrm{Mbps}$ being achieved with 802.11a hardware [3]. However, vehicular traffic scenarios have greater challenges than fixed wireless networks, caused by varying $\mathrm{d}$ speeds of driving, patterns of traffic, and driving environments. Traditional IEEE 802.11 Media Access Control (MAC) operations suffer from significant overheads when used in vehicular scenarios. For instance, to confirm timely vehicular safety communications, fast data exchanges are required. In these circumstances the scanning of channels for beacons from an Access Point along with multiple handshakes required to establish communication are associated with too much complexity and high overheads. To address these challenging requirements of IEEE MAC operations, the DSRC effort of the ASTM 2313 working group migrated to the IEEE 802.11 standard group which changes the name from DSRC to IEEE 802.11p Wireless Access in Vehicular Environments (WAVE) [4].

WAVE defines two types of devices: RoadSide Unit (RSU), and OnBoard Unit (OBU) which are essentially stationary and mobile devices respectively. Both RSUs and OBUs can be either a provider or a user of services and can switch between such modes. Usually stationary WAVE devices host an application that gives service, and the mobile device which hosts a peer application that uses such a service. There may also be applications on devices remote from the RSU whose purpose is to give services to the OBU. This WAVE standard defines applications that resides on the RSU but is designed to multiplex requests from remote applications thus providing them with access to the OBU.

\section{LITERATURE SURVEY FOR CLUSTERING PROTOCOLS}

For efficient communication among the nodes in the network, stable clustering is required. In this direction, many researchers have used various techniques to form a stable cluster among the nodes. Some of these techniques consist of the use of signal strength received, node position from the cluster head, velocity of the nodes and direction and destination of node. Keeping in view of the above issues, the detailed taxonomy of various clustering algorithm is described in Fig.5.

\subsection{Predictive Clustering}

In predictive clustering, the cluster structure is determined by the current geographic position of vehicles and its future behavior. This vehicle traffic information helps to associate priorities which then assist in cluster formation. The future position and the intended destinations of vehicles have been used in the literature to form clusters in VANETs. Some of these protocols are classified as position based and destination based as follows:

\subsubsection{Position Based Clustering}

Position based clustering is a technique of forming the clusters on the basis of geographic position of the vehicle and cluster head.

Salhi et al. [5] proposed a new position based clustering algorithm (NEW-ALM) which is an improvement to the existing ALM algorithm. 
Wang et al. [6] proposed another position based clustering algorithm. It is a cross layer algorithm based on hierarchical and geographical data collection and dissemination mechanism.

Fan et al. [7] proposed a clustering scheme where a utility based cluster formation technique is used by extending the definition of Spatial Dependency which was initially proposed in []].

Maslekar et al. [9] proposed a new cluster-head election policy for direction based clustering algorithm called as Modified Clustering based on Direction in Vehicular Environment (MC-DRIVE) [10].

Wolny [11] optimized the existing DMAC algorithm presented in [12] so that road traffic mobility is represented in an efficient manner.

\section{Discussion on Position Based Clustering}

Since the above clustering protocols primarily rely on the vehicle position, the range of values for vehicular density and vehicle speed exhibits a variation for every protocol. However the value of cluster convergence rate is low even if vehicle density and cluster dynamics increase which points to better cluster stability for these schemes. The variation in cluster size also affects performance in terms of mean cluster diameter and dismiss threshold for position based clustering. The value of transmission efficiency

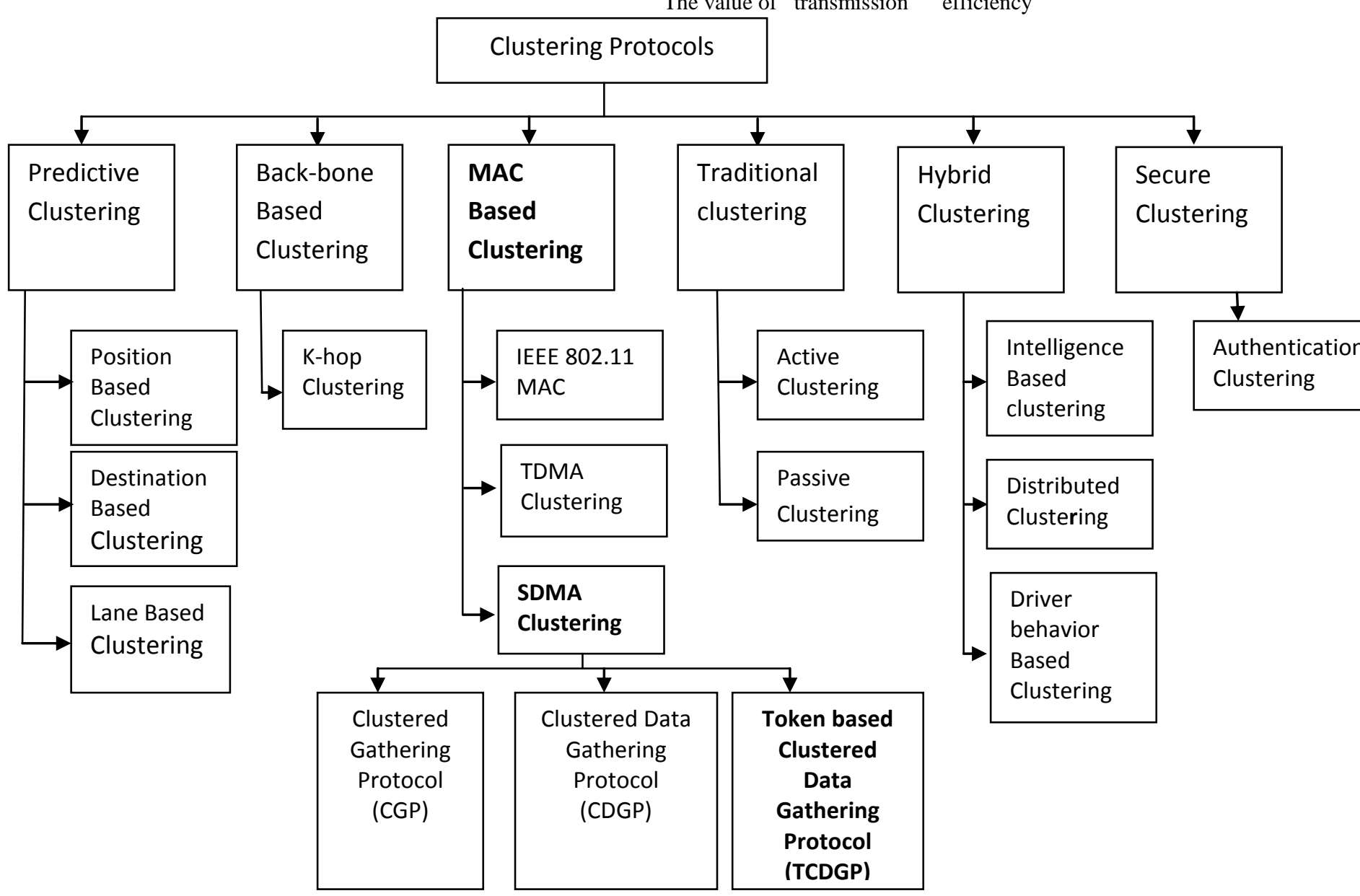

Fig. 5: Taxonomy of existing clustering approaches for VANeT

which ultimately effects packet delivery ratio is also on an average on the lower side. It can be concluded that transmission overhead and cluster connect time needs further analysis for improving the overall efficiency of clustering.

\subsubsection{Destination Based Clustering}

Destination based clustering technique takes into account the current position, speed, relative and final destination of vehicle for cluster formation. Using the navigation system the destination is known previously. Various proposals in this category are described as follows:

Farhan et al. [13] proposed an algorithm for improving the accuracy of GPS devices called Location Improvement with Cluster Analysis (LICA)
Tian et al. [14] presented a clustering method based on a vehicles position and moving direction. The clustering method is based on Euclidean distance, which uses the position information as well as the moving direction to divide the vehicles into clusters. Each vehicle broadcasts beacon message that include its ID latitude, longitude, direction and time to the whole network. Adaptable Mobility-Aware Clustering Algorithm based on Destination (AMACAD) [15] is based on final destination in vehicular networks to enhance the clustering stability.

Region Group Mobility model proposed in [16] was also modified to make it suitable for VANets.

In AMACAD, the authors evaluated how the variation of the trans-mission range and speed affects the AMACAD performance. The algorithm works well when average speed 
of vehicles is almost constant which is most effective in urban areas.

Santos et al. [17] proposed Cluster Based Location Routing (CBLR) algorithm to choose CHs in VANETs. This algorithm is based on the regular transmission of beacons, which are used to distribute the state of the nodes.

\section{Discussion on Destination Based Clustering}

In order to keep the clustering process stable, the frequency of cluster changes is minimized because a vehicle only leaves a cluster when it encounters a $\mathrm{CH}$ whose destination is more similar compared to destination of current $\mathrm{CH}$. Thus exploiting the vehicular behavior by taking into account the final destinations of vehicles enhances the cluster stability and improves the transmission efficiency in message delivery. It also results in higher cluster connect time as the probability of a vehicle leaving a cluster is generally low due to similarity in their destinations. However in case the number of vehicles in a cluster becomes large message broadcast results in high transmission overhead.

\subsubsection{Lane Based Clustering}

Lane based clustering forms the cluster structure based estimation of vehicles lane with respect to certain parameters. Some of the proposals in this category are explained as follows:

Fan et al. [18] proposed Broadcasting based Distributed Algorithm (BDA) to stabilize the existing clusters that require only single hop neighbor knowledge and incur minimal overhead. This approach attempts to improve the performance of classical clustering algorithms by making them aware of the vehicle's movement.

Almalag et al. [19] presented a lane-based clustering algorithm based on the traffic flow of vehicles. The proposed algorithm is based on the assumption that each vehicle knows its exact lane on the road through some lane detection system and in depth dig-ital street map that includes lane information. It also uses GPS combined with wheel odometer for lane detection of a vehicle.

The authors use the same general idea as the utility algorithm in [18] , but apply a different set of rules. Each vehicle computes and broadcasts its Cluster Head Level (CHL) along with its speed and other parameters.

\section{Discussion on Lane Based Protocols}

Lane based clustering algorithms use the availability of lane in-formation to select stable clusters. It indicates that the above two schemes have low number of $\mathrm{CH}$ changes that improves the cluster stability. The transmission overhead of these schemes is also reasonable on account of small number of retransmissions of broadcast messages since re-clustering is performed only at lane intersections. These schemes also display improved transmission efficiency due to better broadcasting reachability and good cluster head lifetime as the vehicles in the same lane move with almost constant relative speed that results in highly stable cluster dynamics. These schemes also exhibit small delay overhead that demonstrates their usefulness for maintaining the cluster even for high mobility vehicular networks. The cluster connect time for these clustering schemes is also reasonable. Thus both the lane based clustering schemes exhibit good clustering characteristics with the constraints that placement of the vehicles should be on the same lane. The observed values of vehicle characteristics such as density and speed is on the lower scale since these protocols are adapt-able for urban environment due the constraint of vehicle travelling in the same lane.

\subsection{Backbone Based Clustering}

Backbone based clustering technique is based on forming a backbone for cluster communication. The backbone then performs the communication and assists in $\mathrm{CH}$ election among the members of the cluster. Many backbones based clustering techniques in this are classified as follows:

\subsection{1 k-hop Clustering}

In multi hop or k-hop clustering, cluster structure is controlled by the hop distance. Every cluster has one of the nodes in the cluster as the $\mathrm{CH}$. The distances between a $\mathrm{CH}$ and the members of the cluster are within a predetermined maximum number of hops which can be one or more hops. Some of the research proposals in this category are explained as follows:

Zhang et al. [20] proposed a multi-hop clustering scheme based on the mobility metric for representing N-hop mobility. A vehicle is allowed to broadcast beacon message periodically and a vehicle calculates Relative Mobility based upon two consecutive beacon messages received from the same node in $\mathrm{N}$ hop distance. Each vehicle node then calculates the aggregate mobility value, which is the sum of relative mobility values into weight value for all the neighboring nodes in N-hops. The vehicle nodes then broadcast their aggregate mobility value in the N-hop neighborhood and the vehicle with smallest aggregate mobility value is selected as the $\mathrm{CH}$ and the other vehicle nodes work as cluster member nodes.

Zhang et al. [21] proposed a novel k-hop clustering approach that takes into account the highest connectivity, vehicle mobility and host ID to select $\mathrm{CH}$. The proposed

clustering approach modifies max-min k-hop heuristic approach defined in [22] for cluster formation by considering highest connectivity in terms of signal strength and vehicle mobility.

Wei et al. [23] proposed a robust Criticality-based Clustering Algorithm (CCA) for VANETs that employed network criticality to direct the process of building clusters.

Dror et al. [24] proposed a distributed randomized two hop clustering algorithm and named as Hierarchical Clustering Algorithm (HCA) that was influenced by the work presented in [25].

\section{Discussion on K-hop Clustering Protocols}

To improve clustering efficiency, multi-hop clustering algorithm uses pros of the k-hop algorithms. This clustering scheme has the benefits of better cluster stability as well as low cluster dynamics and can be attributed to the reduced variation in $\mathrm{CH}$ and cluster-member life time. Thus it is concluded that this clustering scheme provide reliable and improved performance of VANets. However impact of speed of vehicle and behavior of vehicle density need to be further analyzed.

\subsection{MAC Based Clustering}

Several Medium Access Control (MAC) based clustering techniques have been proposed for cluster formation in VANETs. These techniques use IEEE 802.11 MAC protocol to generate clusters. Some of popular MAC based protocols are discussed as follows 


\subsubsection{IEEE 802.11 MAC Based Clustering}

$\mathrm{Su}$ et al. [26] proposed a cluster based Multichannel communication scheme that integrates Clustering with MAC protocols (CB-MMAC). The proposed scheme mainly consists of three core protocols called Cluster Configuration Protocol that groups all vehicles in the same direction into clusters.

Bonini et al. [27] proposed a cross-layered clustering scheme for fast propagation of broadcast messages which is called as Dynamic Backbone Assisted MAC (DBA-MAC) scheme that may be considered an extension of the MAC scheme described in [28].

\section{Discussion on IEEE 802.11MAC Based Protocols}

MAC based protocols have increased percentage collisions and average message delivery delay that results in lower transmission efficiency and high transmission overhead due to increased contention when number of vehicles or speed of the vehicle increases. Liu et al. [29] identified message delivery distance and density of vehicles as two main factors for such behavior based on a bidirectional vehicle traffic model. The consideration of bi-directional traffic also affects cluster connect time and results in lower cluster convergence. However in these proto-cols the percentage collisions and incurred overhead for delivering safety messages is decreased by reducing channel contentions for achieving timely and reliable delivery of safety messages.

\subsubsection{TDMA Based Clustering}

The process of assigning time slots can be scheduled using TDMA technique in which slots are assigned for data transmission. Some of the proposals in this category are described as follows:

Biswas et al. [30] proposed Vehicular Self-Organized MAC (VeSOMAC) protocol based on a self-configuring TDMA slot reservation protocol which is capable of inter-vehicle message delivery with short and deterministic delay bounds.

Omar et al. [31] proposed a multichannel MAC protocol for VANETs, called VeMAC, to reduce interference between vehicles and reduce transmission collisions caused by vehicle mobility. VeMAC is based on a TDMA scheme for intervehicle communication.

Gunter et al. [29] proposed cluster based medium access control protocol (CBMAC), where the $\mathrm{CH}$ takes on a managerial role and facilitates intra-cluster communication by providing a TDMA schedule to its cluster members. The CBMAC protocol uses an adoption of CBLR protocol proposed in [15] for cluster formation.

Almalag et al. [32] proposed a new TDMA Cluster-based MAC (TC-MAC) that can be used for intra-cluster communications in VANETs. This protocol integrates the centralized approach of cluster management and a new scheme for TDMA slot reservation.

\section{Discussion on TDMA Based Clustering}

The access to the medium within a cluster is based on TDMA which is primarily used for optimizing communication. These clustering protocols reduce intra cluster collisions as well as packet loss compared to traditional clustering protocols and thus provide fairness in sharing the wireless medium for VANETs. It shows that TDMA algorithms have relatively smaller delay of multi-hop safety messages as compared to other clustering schemes. Thus they provide better transmission efficiency for cluster maintenance which improves the overall throughput of both inter-cluster and intra-cluster communication. Although cluster connect time is comparatively reasonable, but high clustering convergence due to TDMA time slot is a serious bottle-neck in implementing these protocols in VANTE's.

\subsubsection{SDMA Based Clustering}

In SDMA based protocols, the road is subdivided into fixed length segments, and a segment is again divided into a fixed number of blocks. Each block is assigned a timeslot representing the al-lowed time for a vehicle to transmit data. SDMA is known to have better performance in a

dense network where practically all slots are used. But, the performance decreases proportionally with the density. Hence, in sparse networks, SDMA gives poor performance.

\subsubsection{Clustering Gathering Protocol (CGP)}

Salhi et al. [33] proposed a protocol for hybrid vehicular architecture, called Clustered Gathering Protocol (CGP). The protocol is designed to provide real-time data (e.g. average speed of vehicle) related to speed of vehicle etc. to base station.

Chang et al. [34, 35] proposed a different dynamic cluster based vehicle to vehicle protocol using SDMA. The protocol was called

Traffic Gather. This protocol inherits all the drawbacks of the use of a static medium access technique in wired or sensors networks. Thus, in the case of sparse density, many allowed slots will not be utilized.

\subsubsection{Clustered Data Gathering Protocol (CDGP)}

Brik et al. [36] proposed a new data collection protocol for vehicular environments called Clustered Data Gathering Protocol (CDGP). The use of a clustering technique in hybrid architecture, Dynamic SDMA in the data collection phase and retransmission mechanism to deal with erroneous data is the major characteristics of CDGP.

\section{Discussion on SDMA-Based Clustering Protocols}

In the above paragraph, the authors have proposed a Robust Clustered Data Gathering Protocol (CDGP), by using a Dynamic Space Division Multiple Access (D-SDMA) technique with a retransmission mechanism. Despite the large number of unused slots, which are wasted in a V2V communication, CDGP provides low collection efficiency. To improve the data collection efficiency a new data collection protocol using an enhanced Dynamic SDMA technique are come into existence called token based cluster data gathering protocol(TCDGP).

\subsubsection{Token based Clustered Data Gathering Protocol (TCDGP):}

Brik et al. [37] proposed an improved data collection protocol called Token-based CDGP (TCDGP) which minimizes the number of lost slots and enhances the efficiency of data collection. TCDGP inherits all the advantages presented by CDGP, with an additional feature that provides reliable and robust data collection.

This protocol will be discussed in detail later, as it is the main emphasis of this paper.

\subsection{Traditional Clustering}

This section discusses the Traditional Clustering techniques used in VANETs. These techniques are further divided in to 
active and passive clustering based upon the role of nodes in VANET

\subsubsection{Active Clustering}

In case of active clustering protocols, there are continuous updates of the clustering information and routing table for route discovery after a fixed interval of time. They generally initiate clustering process through flooding which generates a sustained routing overhead. The various Active Clustering protocols are: Beacon based clustering, Mobility based clustering, Density based clustering, Dynamic clustering.

\subsubsection{Passive Clustering}

Passive clustering is a clustering mechanism that passively constructs a cluster structure $[21,56]$. At any time, anode in a cluster possesses an external or internal state. In passive clustering each vehicle can lower the control overhead in packet flooding by the use of on-going data packets instead of extra explicit control packets to construct and maintain the clusters. When a node receives data packets, its cluster state may change, based on the state information piggybacked in on-going data packets. This reduces the number of explicit control packets. Thus Passive clustering mechanism generates significantly less overhead for cluster maintenance than the traditional cluster-based technique because its nodes do not maintain cluster information all the time.

\subsection{Hybrid Clustering}

Hybrid clustering techniques combine two or more existing techniques such as use of artificial intelligence, fuzzy logic etc. Following are the schemes in this category of clustering.

\subsubsection{Intelligence Based Clustering}

Hafeez et al. [38] proposed a distributed and dynamic Cluster head selection criteria to organize the network into clusters. $\mathrm{CH}$ is elected based on stability criteria which reflect the relative movement between adjacent vehicles.

Kumar et al. [39] proposed an Agent Learning-based Clustering Algorithm (ALCA). Agents are able to learn from the environment in which they are operating and perform the task of $\mathrm{CH}$ selection. The proposed approach consists of selection of $\mathrm{CH}$ keeping in view of the direction of mobility and density of the nodes.

\section{Discussion on Intelligence Based Clustering Protocols}

All the intelligence based clustering protocols have good cluster stability which is due to large $\mathrm{CH}$ duration and cluster member duration. The value of these parameters also improves as the vehicle density increases. These protocols generate reasonably stable clusters but they also cause large transmission overhead which reduces the packet delivery ratio resulting in reduced transmission efficiency for the discussed protocols.

\subsubsection{Cooperative De-centralized Clustering}

Cooperative vehicular systems are currently being investigated to design innovative ITS solutions for road traffic management and safety. Through various wireless technologies, cooperative systems can support novel decentralized strategies for ubiquitous and cost effective traffic monitoring system [40].

QuickSilver [41] is a light weight distributed clustering protocol that integrates a traditional source routing protocol for intra cluster node centric communication and the construction of a multichannel link for contention free inter cluster data centric communication. It is a system architecture that provides efficient use of available resources to guarantee that no harmful competition takes place for the channel bandwidth.

\section{Discussion on Cooperative De-centralized Clustering}

These clustering protocols have low cluster stability and average cluster connect time. This is due to the fact that average number of inter-cluster links that are active when vehicles are in contact initially increases as the overlapping region for a vehicle increases and then it shows a corresponding decrease as vehicles move away from each other. Transmission efficiency also has comparable value for these protocols. Thus high transmission overhead and lower vehicle density results in reduced effectiveness for intracluster communication in spite of decentralized clustering schemes considering realistic vehicular speed conditions.

\subsubsection{Driver behavior Based Hybrid Clustering}

Vehicles are nowadays provided with a variety of sensors capable of gathering information from their surroundings. In near future, these vehicles will also be capable of sharing all the harvested information, with the nearby environment and among surrounding vehicles over smart wireless links. in case of accidents, they will also be able to connect with emergency services [42].

Blum et al. [43] proposed a Clustering for Open Inter vehicle communication Networks (COIN) algorithm. In COIN, $\mathrm{CH}$ election is based on vehicular dynamics and driver intentions as input for clustering instead of any conventional parameter like vehicle ID, relative mobility or some other parameters that are used in a classical clustering method. Further COIN attempts to preserve $\mathrm{CH}$ for a longer duration and uses mobility information for clustering.

Cheng et al. [44] proposed an innovative car-society clustered network based on an imaginative classification scheme. The proposed scheme forms clusters by including vehicles that have the same interest and operating in the same communication range. The aim of the proposed approach is to increase the lifetime of the interest group, and to increase throughput in $\mathrm{V} 2 \mathrm{~V}$ environments.

\section{Discussion on Driver behavior Based Hybrid Clustering Protocols}

Driver Intention based Hybrid clustering protocols improve the effectiveness of clustering in terms of cluster lifetime of same interest groups of users that shows good cluster stability. The transmission efficiency for these protocols is also comparable with other clustering schemes but these may have generated for lower values of vehicular speed and vehicle densities. This also results in satisfactory transmission overhead and cluster dynamics which may however not happen if realistic vehicular conditions are considered. Clustering Convergence and Cluster Connect Time also need further investigations. Thus although these protocols provide adequate stability in terms of lifetime of same interest groups but they also need to be further analyzed on the basis of several other parameters for considering their suitability in vehicular environments.

\subsection{Secure Clustering}

VANETs can support applications and services for safety and comfort for the passengers on the road and assist in improving the efficiency of the road transportation network. However, many serious challenges remain to be solved before efficient and secure VANET technology becomes available. One of these challenges is an efficient authentication of messages using cryptographic techniques [45]. Solutions for secure 
clustering in VANETs require efficient clustering algorithms in terms of complexity, scalability, availability and reach ability.

\subsubsection{Authentication Clustering}

Several algorithms have been proposed in the literature based on Public Key Infrastructure (PKI) for enabling communications security in vehicular environments. These are based on a trusted third party called as Certification Authority (CA) which is responsible for certifying the public keys of vehicles.

Blum et al. [46] used a PKI with virtual infrastructure where a set of elected CHs are responsible for disseminating messages after digitally signing them. This scheme is intended only for the attack called intelligent collisions. However, a PKI in VANETs must cope with different attacks

Raya et al. [47] proposed a distributed PKI for VANETs managed by many certification authorities, each corresponding to a particular region. The different CAs has to be cross-certified so that vehicles from different regions can authenticate each other's CA.

Sivagurunathan et al. [48] proposed a self-organized key management system based on clustering. In their model, the network is divided into number of clusters based on the concept that any user can sign any other public key. The set of signatures forms the network of trusted relationships.

Gazdar et al. [49] proposed an efficient dynamic architecture of PKI for VANETs based on a trust model. Each elected vehicle will be the $\mathrm{CA}$ in its cluster. The proposed clustering algorithm is based on a Trust Metric (Tm) which defines the trust level of a vehicle and is a continuous value in the interval [0-1] and mobility metric which is the relative velocity of a vehicle related to its vicinity.

\section{Discussion on Secure Clustering Protocols}

Secure clustering is an approach to increase the channel transmission efficiency and to decrease delay through authentication of a vehicle or a message. These protocols also provide higher level of security which provides data correctness and higher number of message broadcasts that increases the transmission overhead.

\section{TOKEN BASED CLUSTERED DATA GATHERING PROTOCOL (TCDGP)}

In this section we describe our new data collection protocol for vehicles on the highways: TCDGP. As mentioned earlier, TCDGP inherits the two major characteristics from CDGP: (i) the use of a clustering technique in V2V and V2I architectures, and (ii) the use of a retransmission mechanism in the case of erroneous data. Moreover, to minimize the number of lost slots and the waiting time of vehicles, we propose a new Token based Dynamic SDMA (TD-SDMA) technique which will be used in the local data collection phase.

For a proper execution of all phases, which make up TCDGP, we make the following assumptions:

1. All vehicles are equipped with GPS device for localization.

2. To know the positions of the RSUs on the highway, each vehicle is equipped with an embedded digital road map.

3. All the RSUs are interconnected on the highway to ensure the return of the data collected to the RSU, which initiated the collection process.

\section{TCDGP Overview}

The execution of TCDGP and its operational steps are illustrated in Fig. 6 and explained as follows:

The step (1) in the algorithm is the collection process initiation phase, in which an RSU initiates the data collection process. In step (2) the collection area is partitioned into two types of virtual segments with the same length: Collection Segments (CS) and Silence Segments (SS). Then, each CS is divided into several blocks with the same length in order to apply the proposed technique TD-SDMA. The step (3) is the cluster head $(\mathrm{CH})$ election phase that allows a set of vehicles in a CS, to decide which one of them will collect the information in the next phase. Finally, in step (4), each $\mathrm{CH}$ collects data from all the vehicles in its CS by using TDSDMA technique. It is to note that the two last phases start and execute periodically in alternation.

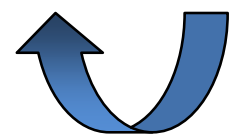

Fig. 6 TCDGP Overview

\subsection{Collection Process Initiation Phase}

When the collection process initiation phase starts, the RSU informs all vehicles on a predetermined area of the highway, of the beginning of the collection process. It therefore broadcasts a Col packet containing essentially the length of the collection area (A-length), the Direction of the Collection (DC), the Data Type (DT) and the Validation Time (VT) of the message

\subsection{Collection Area and Segment Division Phase}

While the data collection process is running, informed vehicles forms several clusters by dividing the area collection into several segments.

\subsubsection{Collection Area Division}

The collection area designated by the initiator is divided into several virtual segments with the same length Ls. In addition, we define two types of segments: Collection Segments (CS), where vehicles can communicate with each other and Silence Segments (SS), where no communication is permitted, in order to avoid collision problems between adjacent segments during V2V communication (Fig.6).

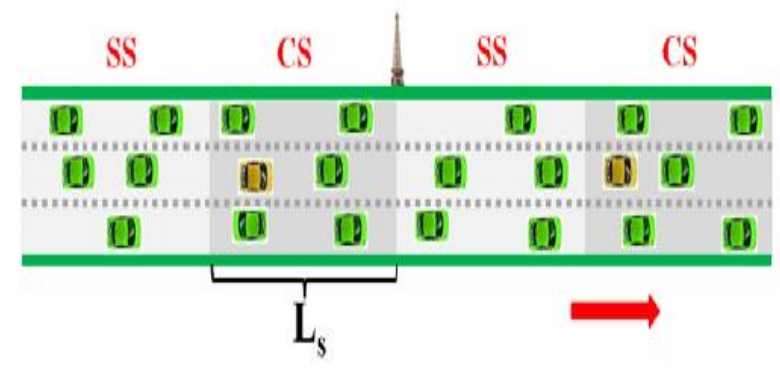

Fig.7: Collection area segmentation.

\subsection{Cluster Head Election Phase}

For the cluster head election phase, we use the back-off based algorithm proposed in [36]. We select a vehicle as a $\mathrm{CH}$ according to two criteria: The closest vehicle to the center of the CS and the efficient vehicle that has circulated more on the middle lanes of the road. This allows us to extend the 
lifetime of the $\mathrm{CH}$ in the $\mathrm{CS}$ and avoid fast reelections of a newer cluster heads.

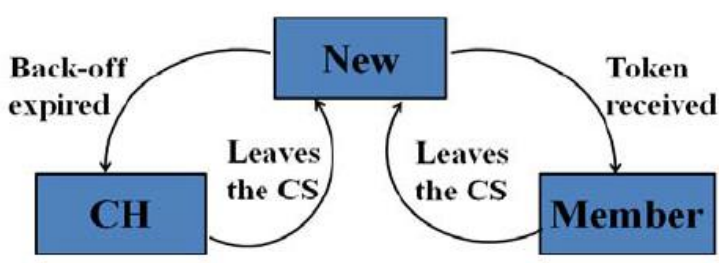

Fig. 8: State machine for the $\mathrm{CH}$ election.

Data collection is based on the use of a token ring, where each $\mathrm{CH}$ generates a Token packet and sends it to each block in its CS. To avoid collision between vehicles in the same CS, we use SDMA technique, where a time slot is assigned to each block in the CS.

As TCDGP aims at minimize the waiting time and at improving the efficiency and reliability of data collection, we propose a new mechanism based on SDMA. This technique allocates a number of slots corresponding to the number of active vehicles in the CS and that has data to send. We called this technique Token-based Dynamic SDMA (TD-SDMA). In TD-SDMA each $\mathrm{CH}$ periodically sends a Token packet that comprises essentially two fields, Block-num: the block number intended by this package and, the Ack field, a single bit, which is used to acknowledge the receipt of data in order to retransmit erroneous data.

Moreover, to allocate time slots to vehicles and inform them of erroneous data, each $\mathrm{CH}$ uses two vectors:

RSV (T-bits): Each bit of this array corresponds to a block on the CS. Thus, a vehicle on a block is assigned a time slots if and only if its corresponding bit in RSV vector is set to 1 .

Ack (T-bits): Used by the CHs to acknowledge the receipt of Data packets and implement the retransmission mechanism.

\subsection{Local Data Collection Phase}

\subsubsection{Data Collection by the ClusterHead}

Following the election phase, each $\mathrm{CH}$ on a CS must collect data from all active vehicles members and inform them of retransmission if it is deemed necessary. To do this, each elected $\mathrm{CH}$ executes various iterations. During the first iteration the reservation of time slots is static, i.e. the $\mathrm{CH}$ assigns a time slot to each block of its CS. So, the

$\mathrm{CH}$ generates a Token packet and sends it to the first block of its CS. Then, it waits to receive a Data packet for a sixth time slot. If it does not receive data during this time, it regenerates a new Token, it sends it to the next block, so on until the last block of the CS. Along with this, it reserves both tables RSV and Ack based on the Data packets received from MVs.

In the iterations other that the initial one, each $\mathrm{CH}$ assigns a time slot by sending a Token packet to each block which its corresponding bit in the RSV array is set to 1. Furthermore, the New Arrivals from vehicles (NA) are allowed fixed number of slots equal to the number of lanes.

\subsubsection{Data Sending by the Member Vehicle}

To deal with the high mobility of vehicles and avoid the change of block by a MV during the transfer, and before sending a Data packet, each MV saves its current block number. When it receives a Token packet, it checks the field
Block-num in the Token whether it corresponds to its old block number or not before checking Ack field.

\subsubsection{Efficiency and Robustness of TCDGP}

To ensure fairness between vehicles and provide efficient data collection, we propose to use TD-SDMA through the cluster heads in the collection segments. Our technique avoids collisions problems and allows a dynamic allocation of slots. In addition, it enhances the reliability by integrating it to the retransmission mechanism. Moreover, the use of More Data (MD) field in Data packet also improves the collection efficiency by reserving a time slot for only the vehicles having data to send.

Unlike our previous protocol CDGP where the entire duration of slot will be lost if a $\mathrm{CH}$ allocates a time slot to an empty block, the worst case for wasted time in TCDGP is only sixth of a time slot. This is so since each $\mathrm{CH}$ has to wait a sixth time slot before generating and sending a new token packet to the next block. These results in minimizing the waiting time of vehicles on CS and enhance the efficiency of data collection process.

\section{CONCLUSION AND FUTURE WORK}

In recent years VANets becomes widely used promulgation technology. The research association has been examined the clustering of vehicles from different aspects in various application used in VANets. This survey paper provides complete characterization of existing clustering protocols with its pros and cons and also discussed the current running clustering protocol called token based clustered data gathering protocol with new features like avoiding collision problems and providing efficient data collection using dynamic medium access technique based on the use of token ring. In future, this works intends to prove the correctness of TCDGP and to adapt it to urban environment.

\section{REFERENCES}

[1] Sun, J., C. Zhang, and Y. Fang. An id-based framework achieving privacy and non-repudiation in vehicular ad hoc networks. in Military Communications Conference, 2007. MILCOM 2007. IEEE. 2007. IEEE.

[2] Stampoulis, A. and Z. Chai, A survey of security in vehicular networks. Project CPSC, 2007. 534.

[3] Committee, L.M., IEEE Std 802.11-2007: IEEE Standard for Information TechnologyTelecommunications and Information Exchange between Systems-Local and Metropolitan Area Networks-Specific Requirements Part 11: Wireless LAN Medium Access Control (MAC) and Physical Layer (PHY) Specifications. IEEE Computer Society, 2007.

[4] Jiang, D. and L. Delgrossi. IEEE 802.11 p: Towards an international standard for wireless access in vehicular environments. in Vehicular Technology Conference, 2008. VTC Spring 2008. IEEE. 2008. IEEE.

[5] Salhi, I., M. Cherif, and S. Senouci. Data collection in vehicular networks. in Proc. ASN symposium. 2007.

[6] Wang, Z., et al., A position-based clustering technique for ad hoc intervehicle communication. Systems, Man, and Cybernetics, Part C: Applications and Reviews, IEEE Transactions on, 2008. 38(2): p. 201-208.

[7] Fan, W., et al., A mobility metrics based dynamic clustering algorithm for VANETs. 2011. 
[8] Bai, F., N. Sadagopan, and A. Helmy, The IMPORTANT framework for analyzing the Impact of Mobility on Performance Of RouTing protocols for Adhoc NeTworks. Ad Hoc Networks, 2003. 1(4): p. 383-403.

[9] Maslekar, N., et al. Modified C-DRIVE: Clustering based on direction in vehicular environment. in Intelligent Vehicles Symposium (IV), 2011 IEEE. 2011. IEEE.

[10] Maslekar, N., et al. A stable clustering algorithm for efficiency applications in VANETs. in Wireless Communications and Mobile Computing Conference (IWCMC), 2011 7th International. 2011. IEEE.

[11] Wolny, G. Modified DMAC clustering algorithm for VANETs. in Systems and Networks Communications, 2008. ICSNC'08. 3rd International Conference on. 2008. IEEE.

[12] Basagni, S. Distributed clustering for ad hoc networks. in Parallel Architectures, Algorithms, and Networks, 1999.(I-SPAN'99) Proceedings. Fourth InternationalSymposium on. 1999. IEEE.

[13] Ahammed, F., J. Taheri, and A. Zomaya. LICA: robust localization using cluster analysis to improve GPS coordinates. in Proceedings of the first ACM international symposium on Design and analysis of intelligent vehicular networks and applications. 2011. ACM.

[14] Tian, D., et al. A VANETs routing algorithm based on Euclidean distance clustering. in Future Computer and Communication (ICFCC), 2010 2nd International Conference on. 2010. IEEE.

[15] Morales, M.M.C., C.S. Hong, and Y.C. Bang. An adaptable mobility-aware clustering algorithm in vehicular networks. in Network Operations and Management Symposium (APNOMS), 2011 13th AsiaPacific. 2011. IEEE.

[16] Zhang, Y., J.M. Ng, and C.P. Low, A distributed group mobility adaptive clustering algorithm for mobile ad hoc networks. Computer Communications, 2009. 32(1): p. 189-202.

[17] Santos, R., R. Edwards, and N. Seed. Inter vehicular data exchange between fast moving road traffic using an ad-hoc cluster-based location routing algorithm and 802.11 b Direct Sequence Spread Spectrum radio. in PostGraduate Networking Conference. 2003.

[18] Almalag, M.S. and M.C. Weigle. Using traffic flow for cluster formation in vehicular ad-hoc networks. in Local Computer Networks (LCN), 2010 IEEE 35th Conference on. 2010. IEEE.

[19] Fan, P. Improving broadcasting performance by clustering with stability for inter-vehicle communication. in Vehicular Technology Conference, 2007. VTC2007Spring. IEEE 65th. 2007. IEEE.

[20] Zhang, Z., A. Boukerche, and R. Pazzi. A novel multihop clustering scheme for vehicular ad-hoc networks. in Proceedings of the 9th ACM international symposium on Mobility management and wireless access. 2011. ACM.

[21] Zhang, L. and H. El-Sayed, A novel cluster-based protocol for topology discovery in vehicular ad hoc network. Procedia Computer Science, 2012. 10: p. 525534.
[22] Camp, T., J. Boleng, and L. Wilcox. Location information services in mobile ad hoc networks. in Communications, 2002. ICC 2002. IEEE International Conference on. 2002. IEEE.

[23] Li, W., A. Tizghadam, and A. Leon-Garcia. Robust clustering for connected vehicles using local network criticality. in Communications (ICC), 2012 IEEE International Conference on. 2012. IEEE.

[24] Dror, E., C. Avin, and Z. Lotker, Fast randomized algorithm for 2-hops clustering in vehicular ad-hoc networks. Ad Hoc Networks, 2013. 11(7): p. 2002-2015.

[25] Fan, P., et al., Cluster-based framework in vehicular adhoc networks, in Ad-hoc, mobile, and wireless networks. 2005, Springer. p. 32-42.

[26] Su, H. and X. Zhang, Clustering-based multichannel $M A C$ protocols for QoS provisionings over vehicular ad hoc networks. Vehicular Technology, IEEE Transactions on, 2007. 56(6): p. 3309-3323.

[27] Bononi, L. and M. Di Felice. A cross layered mac and clustering scheme for efficient broadcast in vanets. in Mobile Adhoc and Sensor Systems, 2007. MASS 2007. IEEE Internatonal Conference on. 2007. IEEE.

[28] Bononi, L., et al., Design and performance evaluation of cross layered MAC and clustering solutions for wireless ad hoc networks. Performance Evaluation, 2006. 63(11): p. 1051-1073.

[29] Liu, Y., et al., The insights of message delivery delay in VANETs with a bidirectional traffic model. Journal of Network and Computer Applications, 2013. 36(5): p. 1287-1294.

[30] Biswas, S., J. Misic, and V. Misic. ID-based safety message authentication for security and trust in vehicular networks. in Distributed Computing Systems Workshops (ICDCSW), 2011 31st International Conference on. 2011. IEEE.

[31] Omar, H.A., W. Zhuang, and L. Li, VeMAC: A TDMAbased MAC protocol for reliable broadcast in VANETs. Mobile Computing, IEEE Transactions on, 2013. 12(9): p. $1724-1736$

[32] Almalag, M.S., S. Olariu, and M.C. Weigle. Tdma cluster-based mac for vanets (tc-mac). in World of Wireless, Mobile and Multimedia Networks (WoWMoM), 2012 IEEE International Symposium on a. 2012. IEEE.

[33] Salhi, I., M.O. Cherif, and S.-M. Senouci. A new architecture for data collection in vehicular networks. in Communications, 2009. ICC'09. IEEE International Conference on. 2009. IEEE.

[34] Chang, W.-R., H.-T. Lin, and B.-X. Chen. Trafficgather: An efficient and scalable data collection protocol for vehicular ad hoc networks. in Consumer Communications and Networking Conference, 2008. CCNC 2008. 5th IEEE. 2008. IEEE.

[35] Bana, S.V. and P. Varaiya. Space division multiple access (SDMA) for robust ad hoc vehicle communication networks. in Intelligent Transportation Systems, 2001. Proceedings. 2001 IEEE. 2001. IEEE.

[36] Brik, B., et al. An efficient and robust clustered data gathering protocol (CDGP) for vehicular networks. in Proceedings of the second ACM international symposium 
on Design and analysis of intelligent vehicular networks and applications. 2012. ACM.

[37] Brik, B., et al. Token-based Clustered Data Gathering Protocol (TCDGP) in vehicular networks. in Wireless Communications and Mobile Computing Conference (IWCMC), 2013 9th International. 2013. IEEE.

[38] Hafeez, K.A., et al. A fuzzy-logic-based cluster head selection algorithm in VANETs. in Communications (ICC), 2012 IEEE International Conference on. 2012. IEEE.

[39] Kumar, N., N. Chilamkurti, and J.H. Park, ALCA: agent learning---based clustering algorithm in vehicular ad hoc networks. Personal and ubiquitous computing, 2013. 17(8): p. 1683-1692.

[40] Bauza, R. and J. Gozálvez, Traffic congestion detection in large-scale scenarios using vehicle-to-vehicle communications. Journal of Network and Computer Applications, 2013. 36(5): p. 1295-1307.

[41] Crepaldi, R., M. Bakht, and R. Kravets. Quicksilver: application-driven inter-and intra-cluster communication in vanets. in Proceedings of the third ACM international workshop on Mobile Opportunistic Networks. 2012. ACM.

[42] Barrachina, J., et al., VEACON: A Vehicular Accident Ontology designed to improve safety on the roads. Journal of Network and Computer Applications, 2012. 35(6): p. 1891-1900.
[43] Blum, J., A. Eskandarian, and L. Hoffman. Mobility management in IVC networks. in Intelligent Vehicles Symposium, 2003. Proceedings. IEEE. 2003. IEEE.

[44] Cheng, S.-T., G.-J. Horng, and C.-L. Chou, Using cellular automata to form car society in vehicular ad hoc networks. Intelligent Transportation Systems, IEEE Transactions On, 2011. 12(4): p. 1374-1384.

[45] Ying, B., D. Makrakis, and H.T. Mouftah, Privacy preserving broadcast message authentication protocol for VANETs. Journal of Network and Computer Applications, 2013. 36(5): p. 1352-1364.

[46] Blum, J. and A. Eskandarian, The threat of intelligent collisions. IT professional, 2004. 6(1): p. 24-29.

[47] Raya, M., A. Aziz, and J.-P. Hubaux. Efficient secure aggregation in VANETs. in Proceedings of the $3 \mathrm{rd}$ international workshop on Vehicular ad hoc networks. 2006. ACM.

[48] Sivagurunathan, S., et al., Authentic vehicular environment using a cluster based key management. European Journal of Scientific Research ISSN, 2009. 36(2): p. 299-307.

[49] Gazdar, T., A. Benslimane, and A. Belghith. Secure clustering scheme based keys management in VANETs. in Vehicular Technology Conference (VTC Spring), 2011 IEEE 73rd. 2011. IEEE. 